
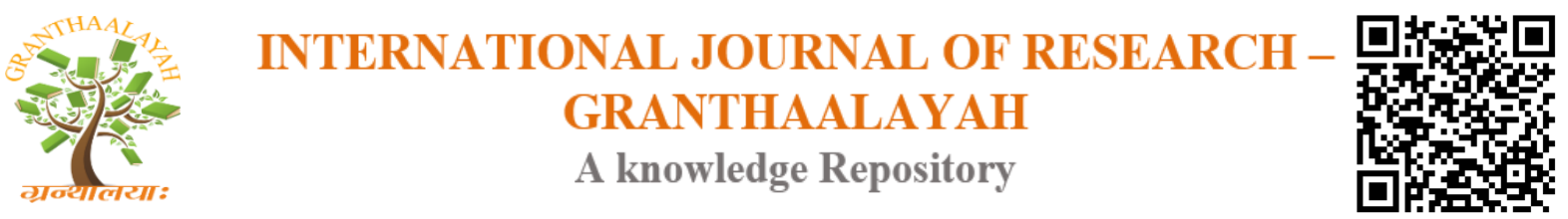

Science

\title{
REAL POWER LOSS MINIMIZATION AND MAXIMIZATION OF STATIC VOLTAGE STABILITY MARGIN BY HYBRIDIZED ALGORITHM
}

\author{
Dr.K.Lenin *1 \\ ${ }^{* 1}$ Professor, Department of EEE, Prasad V.Potluri Siddhartha Institute of Technology, Kanuru, \\ Vijayawada, Andhra Pradesh -520007, India
}

\begin{abstract}
This paper presents a new Hybridized Algorithm (HA) for solving the multi-objective reactive power dispatch problem. Inspired by Genetic Algorithm (GA), Particle Swarm Optimization (PSO) \& the Bat Algorithm (BA), the HA was designed to retain some advantages of each method to improve the exploration and exploitation of the search. Scrutinizing PSO and BA reveals some differences, in that BA rejects the historical experience of each individual's own position but admits an improved personal solution with some probability. We will adjust some of the updating mechanisms of BA and add a mutation method in order to try to solve reactive power problem more accurately. Proposed (HA) algorithm has been tested on standard IEEE 30 bus test system and simulation results shows clearly about the good performance of the proposed algorithm.
\end{abstract}

Keywords: Modal Analysis; Optimal Reactive Power; Transmission Loss; Hybridized Algorithm.

Cite This Article: Dr.K.Lenin. (2017). "REAL POWER LOSS MINIMIZATION AND MAXIMIZATION OF STATIC VOLTAGE STABILITY MARGIN BY HYBRIDIZED ALGORITHM." International Journal of Research - Granthaalayah, 5(7), 506-519. https://doi.org/10.29121/granthaalayah.v5.i7.2017.2159.

\section{Introduction}

Optimal reactive power dispatch problem is subject to number of uncertainties and at least in the best case to uncertainty parameters given in the demand and about the availability equivalent amount of shunt reactive power compensators. Optimal reactive power dispatch plays a major role for the operation of power systems, and it should be carried out in a proper manner, such that system reliability is not got affected. The main objective of the optimal reactive power dispatch is to maintain the level of voltage and reactive power flow within the specified limits under various operating conditions and network configurations. By utilizing a number of control tools such as switching of shunt reactive power sources, changing generator voltages or by adjusting transformer tap-settings the reactive power dispatch can be done. By doing optimal 
adjustment of these controls in different levels, the redistribution of the reactive power would minimize transmission losses. This procedure forms an optimal reactive power dispatch problem and it has a major influence on secure and economic operation of power systems. Various mathematical techniques like the gradient method [1,2] Newton method [3] and linear programming [4-7] have been adopted to solve the optimal reactive power dispatch problem. Both the gradient and Newton methods has the difficulty in handling inequality constraints. If linear programming is applied then the input- output function has to be expressed as a set of linear functions which mostly lead to loss of accuracy. The problem of voltage stability and collapse play a major role in power system planning and operation [8]. Enhancing the voltage stability, voltage magnitudes within the limits alone will not be a reliable indicator to indicate that, how far an operating point is from the collapse point. The reactive power support and voltage problems are internally related to each other. This paper formulates by combining both the real power loss minimization and maximization of static voltage stability margin (SVSM) as the objectives. Global optimization has received extensive research attention, and a great number of methods have been applied to solve this problem. Evolutionary algorithms such as genetic algorithm have been already proposed to solve the reactive power flow problem $[9,10]$.Evolutionary algorithm is a heuristic approach used for minimization problems by utilizing nonlinear and non-differentiable continuous space functions. In [11], by using Genetic algorithm optimal reactive power flow has been solved, and the main aspect considered is network security maximization. In [12] is proposed to improve the voltage stability index by using Hybrid differential evolution algorithm. In [13] Biogeography Based algorithm proposed to solve the reactive power dispatch problem. In [14] a fuzzy based method is used to solve the optimal reactive power scheduling method and it minimizes real power loss and maximizes Voltage Stability Margin. In [15] an improved evolutionary programming is used to solve the optimal reactive power dispatch problem. In [16] the optimal reactive power flow problem is solved by integrating a genetic algorithm with a nonlinear interior point method. In [17] a standard algorithm is used to solve ac-dc optimal reactive power flow model with the generator capability limits .In [18] proposed a two-step approach to evaluate Reactive power reserves with respect to operating constraints and voltage stability. In [19] a programming based proposed approach used to solve the optimal reactive power dispatch problem. In [20] is presented a probabilistic algorithm for optimal reactive power provision in hybrid electricity markets with uncertain loads. This research paper proposes a new Hybridized Algorithm (HA) for solving the multi-objective reactive power dispatch problem.Inspired by Genetic Algorithm (GA), Particle Swarm Optimization (PSO) \& the Bat Algorithm (BA), the HA was designed to retain some advantages of each method to improve the exploration and exploitation of the search. Proposed method HA been evaluated in standard IEEE 30 bus test system \& the simulation results shows that our proposed approach outperforms all reported algorithms in minimization of real power loss and voltage stability index .

\section{Voltage Stability Evaluation}

\subsection{Modal Analysis for Voltage Stability Evaluation}

Modal analysis is one among best methods for voltage stability enhancement in power systems. The steady state system power flow equations are given by. 
$\left[\begin{array}{l}\Delta \mathrm{P} \\ \Delta \mathrm{Q}\end{array}\right]=\left[\begin{array}{cc}\mathrm{J}_{\mathrm{p} \theta} & \mathrm{J}_{\mathrm{pv}} \\ \mathrm{J}_{\mathrm{q} \theta} & \mathrm{J}_{\mathrm{QV}}\end{array}\right]\left[\begin{array}{l}\Delta \theta \\ \Delta V\end{array}\right]$

Where

$\Delta \mathrm{P}=$ Incremental change in bus real power.

$\Delta \mathrm{Q}=$ Incremental change in bus reactive Power injection

$\Delta \theta=$ incremental change in bus voltage angle.

$\Delta \mathrm{V}=$ Incremental change in bus voltage Magnitude

Jp $\theta$, JPV , JQ $\theta$, JQV jacobian matrix are the sub-matrixes of the System voltage stability is affected by both $\mathrm{P}$ and $\mathrm{Q}$.

To reduce (1), let $\Delta \mathrm{P}=0$, then.

$\Delta \mathrm{Q}=\left[\mathrm{J}_{\mathrm{QV}}-\mathrm{J}_{\mathrm{Q} \theta} \mathrm{J}_{\mathrm{P} \theta^{-1}} \mathrm{~J}_{\mathrm{PV}}\right] \Delta \mathrm{V}=\mathrm{J}_{\mathrm{R}} \Delta \mathrm{V}$

$\Delta \mathrm{V}=\mathrm{J}^{-1}-\Delta \mathrm{Q}$

Where

$\mathrm{J}_{\mathrm{R}}=\left(\mathrm{J}_{\mathrm{QV}}-\mathrm{J}_{\mathrm{Q} \theta} \mathrm{J}_{\mathrm{P} \theta^{-1}} \mathrm{JPV}\right)$

$\mathrm{J}_{\mathrm{R}}$ is called the reduced Jacobian matrix of the system.

\subsection{Modes of Voltage Instability}

Voltage Stability characteristics of the system have been identified by computing the Eigen values and Eigen vectors.

Let

$\mathrm{J}_{\mathrm{R}}=\xi \wedge \eta$

Where,

$\xi=$ right eigenvector matrix of JR

$\eta=$ left eigenvector matrix of JR

$\Lambda=$ diagonal eigenvalue matrix of $\mathrm{JR}$ and

$\mathrm{J}_{\mathrm{R}^{-1}}=\xi \wedge^{-1} \eta$

From (5) and (8), we have

$\Delta \mathrm{V}=\xi \wedge^{-1} \eta \Delta \mathrm{Q}$

$\Delta \mathrm{V}=\sum_{\mathrm{I}} \frac{\xi_{\mathrm{i}} \eta_{\mathrm{i}}}{\lambda_{\mathrm{i}}} \Delta \mathrm{Q}$

Where $\xi_{i}$ is the ith column right eigenvector and $\eta$ the ith row left eigenvector of JR.

$\lambda i$ is the ith Eigen value of JR.

The ith modal reactive power variation is,

$\Delta \mathrm{Q}_{\mathrm{mi}}=\mathrm{K}_{\mathrm{i}} \xi_{\mathrm{i}}$

where,

$\mathrm{K}_{\mathrm{i}}=\sum_{\mathrm{j}} \xi_{\mathrm{ij}}-1$

Where

$\xi \mathrm{ji}$ is the jth element of $\xi \mathrm{i}$

The corresponding ith modal voltage variation is $\Delta \mathrm{V}_{\mathrm{mi}}=\left[1 / \lambda_{\mathrm{i}}\right] \Delta \mathrm{Q}_{\mathrm{mi}}$

If $|\lambda \mathrm{i}|=0$ then the ith modal voltage will collapse .

In (10), let $\Delta \mathrm{Q}=\mathrm{ek}$ where ek has all its elements zero except the kth one being 1 . Then, 


$$
\Delta \mathrm{V}=\sum_{\mathrm{i}} \frac{\eta_{1 \mathrm{k}} \xi_{1}}{\lambda_{1}}
$$

$\eta_{1 \mathrm{k}} \quad \mathrm{k}$ th element of $\eta_{1}$ $\mathrm{V}-\mathrm{Q}$ sensitivity at bus $\mathrm{k}$ $\frac{\partial \mathrm{V}_{\mathrm{K}}}{\partial \mathrm{Q}_{\mathrm{K}}}=\sum_{\mathrm{i}} \frac{\eta_{1 \mathrm{k}} \xi_{1}}{\lambda_{1}}=\sum_{\mathrm{i}} \frac{\mathrm{P}_{\mathrm{ki}}}{\lambda_{1}}$

\section{Problem Formulation}

The objectives of the reactive power dispatch problem is to minimize the system real power loss and maximize the static voltage stability margins (SVSM).

\subsection{Minimization of Real Power Loss}

Minimization of the real power loss (Ploss) in transmission lines is mathematically stated as follows.

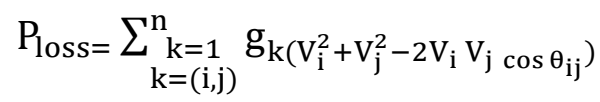

Where $\mathrm{n}$ is the number of transmission lines, gk is the conductance of branch $\mathrm{k}, \mathrm{Vi}$ and $\mathrm{Vj}$ are voltage magnitude at bus $\mathrm{i}$ and bus $\mathrm{j}$, and $\theta \mathrm{ij}$ is the voltage angle difference between bus $\mathrm{i}$ and bus j.

\subsection{Minimization of Voltage Deviation}

Minimization of the voltage deviation magnitudes (VD) at load buses is mathematically stated as follows.

Minimize VD $=\sum_{\mathrm{k}=1}^{\mathrm{nl}}\left|\mathrm{V}_{\mathrm{k}}-1.0\right|$

Where $\mathrm{nl}$ is the number of load busses and $\mathrm{Vk}$ is the voltage magnitude at bus $\mathrm{k}$.

\subsection{System Constraints}

Objective functions are subjected to these constraints shown below.

Load flow equality constraints:

$P_{G i}-P_{D i}-V_{i} \sum_{j=1}^{n b} v_{j}\left[\begin{array}{cc}G_{i j} & \cos \theta_{i j} \\ +B_{i j} & \sin \theta_{i j}\end{array}\right]=0, i=1,2 \ldots, n b$

$Q_{G i}-Q_{D i}-V_{i} \sum_{j=1}^{n b} V_{j}\left[\begin{array}{cc}G_{i j} & \sin \theta_{i j} \\ +B_{i j} & \cos \theta_{i j}\end{array}\right]=0, i=1,2 \ldots, n b$

where, nb is the number of buses, PG and QG are the real and reactive power of the generator, $\mathrm{PD}$ and QD are the real and reactive load of the generator, and Gij and $\mathrm{Bij}$ are the mutual conductance and susceptance between bus $\mathrm{i}$ and bus $\mathrm{j}$.

Generator bus voltage (VGi) inequality constraint:

$\mathrm{V}_{\mathrm{Gi}}^{\min } \leq \mathrm{V}_{\mathrm{Gi}} \leq \mathrm{V}_{\mathrm{Gi}}^{\mathrm{max}}, \mathrm{i} \in \mathrm{ng}$

Load bus voltage (VLi) inequality constraint:

$\mathrm{V}_{\mathrm{Li}}^{\min } \leq \mathrm{V}_{\mathrm{Li}} \leq \mathrm{V}_{\mathrm{Li}}^{\max }, \mathrm{i} \in \mathrm{nl}$ 
Switchable reactive power compensations (QCi) inequality constraint:

$\mathrm{Q}_{\mathrm{Ci}}^{\min } \leq \mathrm{Q}_{\mathrm{Ci}} \leq \mathrm{Q}_{\mathrm{Ci}}^{\max }, \mathrm{i} \in \mathrm{nc}$

Reactive power generation (QGi) inequality constraint:

$\mathrm{Q}_{\mathrm{Gi}}^{\min } \leq \mathrm{Q}_{\mathrm{Gi}} \leq \mathrm{Q}_{\mathrm{Gi}}^{\max }, \mathrm{i} \in \mathrm{ng}$

Transformers tap setting (Ti) inequality constraint:

$\mathrm{T}_{\mathrm{i}}^{\min } \leq \mathrm{T}_{\mathrm{i}} \leq \mathrm{T}_{\mathrm{i}}^{\mathrm{max}}, \mathrm{i} \in \mathrm{nt}$

Transmission line flow (SLi) inequality constraint:

$\mathrm{S}_{\mathrm{Li}}^{\min } \leq \mathrm{S}_{\mathrm{Li}}^{\max }, \mathrm{i} \in \mathrm{nl}$

Where, nc, ng and nt are numbers of the switchable reactive power sources, generators and transformers.

\section{Genetic Algorithm with Laplace Crossover Operator}

The crossover operator is a scheme for producing genetic information from parents; it combines the characters of two parents to form two off-springs, with the possibility that good chromosomes may evaluate better ones. The crossover operator is not regularly imposed to all pairs of parent solution the intermediate generation. An incidental choice is made, where the possibility of crossover being applied depends on probability determined by a crossover rate, known as crossover probability. The crossover operator is most significant part in GAs. It combines portion of good solution to construct new favorable solution. Information involved in one solution mixed with information involved in another solution and the rising solution will either have good quality fitness or stay alive to commutate this information again. If generated two off-springs are the same then crossover operator show strong heritability [21, 22]. Crossover operators play key role in genetic algorithm which combines the characteristic of existing solutions and generate new solutions. The optimization problems depend upon the data they used so they are classified in to two categories. One is based on real data set and another one is based on binary or discrete data set. Crossover operator also considered as binary crossover operators and real coded crossover operators. Two particles distribute their positional information in the search space and a new particle is formed. The particle, is known as laplacian particle, replaces the nastiest performing particle in the swarm. Using this fresh operator, this paper introduces two algorithms namely Laplace Crossover PSO with inertia weight (LXPSO-W) and Laplace Crossover PSO with constriction factor (LXPSO-C) [23]. A. H. Wright suggests a genetic algorithm that uses real parameter vectors as chromosomes, real parameters as genes, and real numbers as alleles [24].Linear crossover [23, 24] is one of the most primitive operator in real coded crossover it develops three solutions from two parents and the best two off-springs substitute parents. Let $\left(x_{1}^{(1, t)}, x_{2}^{(1, t)}, \ldots, x_{n}^{(1, t)}\right)$ and $\left(x_{1}^{(2, t)}, x_{2}^{(2, t)}, \ldots, x_{n}^{(2, t)}\right)$ are two parent solutions of dimension $n$ at generation $t$. Linear crossover develops three offspring from these parents as shown in Eq.(24, 25 and 26) and best two offspring being chosen as off-springs.

$0.5\left(X_{i}^{(1, t)}+X_{i}^{(2, t)}\right)$

$\left(1.5 X_{i}^{(1, t)}-0.5 X_{i}^{(2, t)}\right)$

$\left(-0.5 X_{i}^{(1, t)}+1.5 X_{i}^{(2, t)}\right)$

Where $\mathrm{i}=1,2, \ldots, \mathrm{n}$ 


\section{Particle Swarm Optimization (PSO)}

PSO [25-28] is a population based optimization tool, where the system is initialized with a population of random particles and the algorithm searches for optima by updating generations. Suppose that the search space is $D$-dimensional. The position of the $i$-th particle can be represented by a $D$-dimensional vector $X_{i}=\left(x_{i 1}, x_{i 2}, \ldots, x_{i D}\right)$ and the velocity of this particle is $V_{i}=\left(v_{i 1}, v_{i 2}, \ldots, v_{i D}\right)$. The best previously visited position of the $i$-th particle is represented by $P_{i}=\left(p_{i 1}, p_{i 2}, \ldots, p_{i D}\right)$ and the global best position of the swarm found so far is denoted by $P_{g}=$ $\left(p_{g 1}, p_{g 2}, \ldots, p_{g D}\right)$. The fitness of each particle can be evaluated through putting its position into a designated objective function. The particle's velocity and its new position are updated as follows:

$v_{i d}^{t+1}=\omega^{t} v_{i d}^{t}+c_{1} r_{1}^{t}\left(p_{i d}^{t}-x_{i d}^{t}\right)+c_{2} r_{2}^{t}\left(p_{g d}^{t}-x_{i d}^{t}\right)$

$x_{i d}^{t+1}=x_{i d}^{t}+v_{i d}^{t+1}$

Where $d \in\{1,2, \ldots, D\}, i \in\{1,2, \ldots, N\} N$ is the population size, the superscript $t$ denotes the iteration number, $\omega$ is the inertia weight, $r_{1}$ and $r_{2}$ are two random values in the range $[0,1], c_{1}$ and $c_{2}$ are the cognitive and social scaling parameters which are positive constants.

These both equations are used to update the velocity and position of a particle in the exploration space .The equation (27) is used to balance the search abilities of the particle in the search space. The equation (28) uses the velocity obtained in first equation to get the new position of the particle. Crossover is a Genetic operator which is used after selection in Genetic Algorithm to get the new children using two or more than two parent .It is used to get the healthier solution than current solution. There are various improved version of crossover available to get the value of new-fangled species. Intermingling crossover is also an improved operator which is used to get the new healthier child by using current parent. This operator is applied in PSO to optimize the multi-dimensional function and upsurge the probing capability of the PSO, So that Particle Swarm Optimization optimizes the functions efficiently and did not jammed in the local optima.

\section{Bat Algorithm}

Bat algorithm has been developed by Xin-She Yang in 2010 [29]. Bats use sonar echoes to identify and evade obstacles. They use time delay from emanation to replication and utilize it for navigation. They classically emit short loud, sound impulse and the rate of pulse is usually 10 to20 times per second. Bats are in-bound to frequencies about 20,500 kHz. By execution [31], Pulse rate can be simply determined from range 0 to 1 , where 0 means there is no emanation and by 1 , bats are emitting maximum [30], By utilizing above behavior new bat algorithm can be formulated. Yang [29] used three generalized rules for bat algorithm:

a) All bats use echolocation to sense distance, and they also guess the difference between prey and background barriers in some magical way.

b) Bats fly arbitrarily with velocity $\vartheta_{i}$ at position $x_{i}$ with a fixed frequency $f_{\text {min }}$, varying wavelength $\lambda$ and loudness $A_{0}$ to search for prey. They can automatically adjust the wavelength of their emitted pulses and adjust the rate of pulse emission $r \in[0 ; 1]$, depending on the proximity of their target. 
c) Although the loudness can vary in many ways, we assume that the loudness varies from a large (positive) $A_{0}$ to a minimum constant value $A_{\text {min }}$.

\section{Original Bat Algorithm}

Objective function $f(x), x=\left(x_{1}, \ldots, x_{d}\right)^{T}$

Initialize the bat population $x_{i}$ and $v_{i}$ for $i=1 \ldots n$

Define pulse frequency $Q_{i} \in\left[Q_{\min }, Q_{\max }\right]$

Initialize pulse rates $r_{i}$ and the loudness $A_{i}$

While $(\mathrm{t}<\mathrm{Tmax}) / /$ number of iterations

Generate new solutions by adjusting frequency, and

Updating velocities and locations/solutions

If (rand $(0 ; 1)>$ ri )

Select a solution among the best solutions

Generate a local solution around the best solution

End if

Generate a new solution by flying randomly

If (rand $(0 ; 1)<\mathrm{Ai}$ and $\mathrm{f}(\mathrm{xi})<\mathrm{f}(\mathrm{x})$ )

Accept the new solutions

Increase ri and reduce $\mathrm{Ai}$

End if

Rank the bats and find the current best

End while

Post process results and visualization

The generation of new solution has been performed by moving virtual bats according the following equations:

$\mathrm{Q}_{\mathrm{i}}^{(\mathrm{t})}=\mathrm{Q}_{\min }+\left(\mathrm{Q}_{\max }-\mathrm{Q}_{\min }\right) \cup(0,1)$,

$v_{i}^{(t+1)}=v_{i}^{t}+\left(x_{i}^{t}-\right.$ best $) Q_{i}^{(t)}$,

$x_{i}^{(t+1)}=x_{i}^{(t)}+v_{i}^{(t)}$

Where $\mathrm{U}(0 ; 1)$ is a uniform distribution.

An arbitrary walk with direct exploitation is used for local exploration that modifies the existing best solution according to equation:

$\mathrm{x}^{(\mathrm{t})}=$ best $+\epsilon \mathrm{A}_{\mathrm{i}}^{(\mathrm{t})}(2 \mathrm{U}(0,1)-1)$,

Where $\epsilon$ is the scaling factor, and $A_{i}^{(t)}$ the loudness. The local exploration is launched with the proximity depending on the pulse rate ri and the new solutions accepted with some proximity depending on parameter. In natural bats, where the rate of pulse emission ri increases and the loudness Ai decreases when a bat finds a prey. The above characteristics can be written by the following equations:

$\mathrm{A}_{\mathrm{i}}^{(\mathrm{t}+1)}=\alpha \mathrm{A}_{\mathrm{i}}^{(\mathrm{t})}, \mathrm{r}_{\mathrm{i}}^{(\mathrm{t})}=\mathrm{r}_{\mathrm{i}}^{(0)}[1-\exp (-\gamma \epsilon)]$,

Where $\alpha$ and $\gamma$ and are constants. 


\section{Hybridized algorithm}

The projected Hybridized Algorithm with greedy strategy resembles the outline of the Genetic Algorithm, which can be described as follows: initialization, evaluation, selection, crossover, and mutation. A difference from the Genetic Algorithm is that there is no distinct selection mechanism in the HA, because each individual will generate its offspring by recombination with the global best individual and it does not require an operator to select an individual to evolve. In addition, local search is also employed to increase the algorithm's exploitation capability. Even though Bat Algorithm can solve some tough problems and converge quickly, it frequently cannot evade converging to a local optimum. Scrutinizing PSO and BA reveals some differences, in that BA rejects the historical experience of each individual's own position but admits an improved personal solution with some probability. We will adjust some of the updating mechanisms of BA and add a mutation method in order to try to solve reactive power problem more accurately.

\section{Mutation}

The drive of mutation is to upsurge the diversity of the population and avert them trapping into a local optimum, particularly in the later iterations. So, the probability of mutation will be made low at the beginning and higher later. We set the mutation probability (MP) as follows,

$M P=K * \operatorname{In}\left(\frac{T_{\max }}{T_{\max }-t}\right)$

Where $\mathrm{K}$ is a limiting parameter which can be a constant or a variable, $T_{\max }$ is the maximum number of generations, and $t$ is the current generation.

The mutation formula is given as follows,

$y_{i}^{t}=y_{i}^{t}+\varepsilon * M T$

Where $y_{i}^{t}$ is the solution of an individual after crossover, $\varepsilon \in[-1,1]$ is a uniform random number, and $M T$ is a vector which determines the scope of mutation.

\section{Local Search}

As we know, most of global optimization algorithms have outstanding competence in exploration but are feeble at exploitation. To augment this capability, particularly in the later iterations, we will expect the algorithm to be able to locate the global best rapidly with local search, once it has found the right neighbourhood. The probability of local search will be maintained low in early iterations and elevated later in the search process. The probability of local search will follow the same distribution as mutation (34).

The following formula used for local search,

$$
y_{i}^{t}=y_{*}^{t-1}+\varepsilon * L S
$$


Where $y_{*}^{t-1}$ is the best individual of the current population, $\varepsilon \in[-1,1]$ is a uniform random number, and $L \mathrm{~S}$ is a vector which determines the search scope of the random walk, formulated in the variable space.

Initialize the parameters

Calculate the initialized population

Chose the best individual $y_{*}^{t-1}$

While $\left(t<T_{\max }\right)$

For each individual

Create crossover to produce a new individual $y_{i}^{t}$

If $($ rand $<p$ )

Create mutation for $y_{i}^{t}$

If $($ rand $<p$ )

Create local search for $y_{i}^{t}$

If $f\left(y_{i}^{t}\right)$ is better than $f\left(y_{i}^{t-1}\right)$ then accept the individual

If $f\left(y_{i}^{t}\right)$ is better than $f\left(y_{*}^{t-1}\right)$ then swap $y_{*}^{t-1}$ with $y_{i}^{t}$

Output

\section{Simulation Results}

The efficiency of the proposed HA method is demonstrated by testing it on standard IEEE-30 bus system. The IEEE-30 bus system has 6 generator buses, 24 load buses and 41 transmission lines of which four branches are (6-9), (6-10), (4-12) and (28-27) - are with the tap setting transformers. The lower voltage magnitude limits at all buses are 0.95 p.u. and the upper limits are 1.1 for all the PV buses and 1.05 p.u. for all the PQ buses and the reference bus. The simulation results have been presented in Tables 1, 2, $3 \& 4$. And in the Table 5 shows the proposed algorithm powerfully reduces the real power losses when compared to other given algorithms. The optimal values of the control variables along with the minimum loss obtained are given in Table 1. Corresponding to this control variable setting, it was found that there are no limit violations in any of the state variables.

Table 1: Results of HA - ORPD optimal control variables

\begin{tabular}{|l|l|}
\hline Control variables & Variable setting \\
\hline V1 & 1.049 \\
V2 & 1.043 \\
V5 & 1.042 \\
V8 & 1.038 \\
V11 & 1.001 \\
V13 & 1.037 \\
T11 & 1.00 \\
T12 & 1.00 \\
T15 & 1.01 \\
T36 & 1.01 \\
Qc10 & 2 \\
Qc12 & 2 \\
Qc15 & 3 \\
\hline
\end{tabular}




\begin{tabular}{|l|l|}
\hline Qc17 & 0 \\
Qc20 & 2 \\
Qc23 & 3 \\
Qc24 & 3 \\
Qc29 & 2 \\
Real power loss & 4.2989 \\
SVSM & 0.2469 \\
\hline
\end{tabular}

Optimal Reactive Power Dispatch problem together with voltage stability constraint problem was handled in this case as a multi-objective optimization problem where both power loss and maximum voltage stability margin of the system were optimized simultaneously. Table 2 indicates the optimal values of these control variables. Also it is found that there are no limit violations of the state variables. It indicates the voltage stability index has increased from 0.2469 to 0.2478 , an advance in the system voltage stability. To determine the voltage security of the system, contingency analysis was conducted using the control variable setting obtained in case 1 and case 2. The Eigen values equivalents to the four critical contingencies are given in Table 3. From this result it is observed that the Eigen value has been improved considerably for all contingencies in the second case.

Table 2: Results of HA -Voltage Stability Control Reactive Power Dispatch Optimal Control Variables

\begin{tabular}{|l|l|}
\hline Control Variables & Variable Setting \\
\hline V1 & 1.046 \\
V2 & 1.041 \\
V5 & 1.044 \\
V8 & 1.030 \\
V11 & 1.003 \\
V13 & 1.031 \\
T11 & 0.090 \\
T12 & 0.090 \\
T15 & 0.090 \\
T36 & 0.090 \\
Qc10 & 3 \\
Qc12 & 3 \\
Qc15 & 2 \\
Qc17 & 3 \\
Qc20 & 0 \\
Qc23 & 2 \\
Qc24 & 2 \\
Qc29 & 3 \\
Real power loss & 4.9864 \\
SVSM & 0.2478 \\
\hline
\end{tabular}


Table 3: Voltage Stability under Contingency State

\begin{tabular}{|l|l|l|l|}
\hline Sl.No & Contingency & ORPD Setting & VSCRPD Setting \\
\hline 1 & $28-27$ & 0.1409 & 0.1424 \\
\hline 2 & $4-12$ & 0.1649 & 0.1652 \\
\hline 3 & $1-3$ & 0.1769 & 0.1779 \\
\hline 4 & $2-4$ & 0.2029 & 0.2041 \\
\hline
\end{tabular}

Table 4: Limit Violation Checking Of State Variables

\begin{tabular}{|l|l|l|l|l|}
\hline \multirow{2}{*}{ State variables } & \multicolumn{2}{|c|}{ Limits } & \multirow{2}{*}{ ORPD } & \multirow{2}{*}{ VSCRPD } \\
\cline { 2 - 4 } & Lower & upper & & \\
\hline Q1 & -20 & 152 & 1.3422 & -1.3269 \\
\hline Q2 & -20 & 61 & 8.9900 & 9.8232 \\
\hline Q5 & -15 & 49.92 & 25.920 & 26.001 \\
\hline Q8 & -10 & 63.52 & 38.8200 & 40.802 \\
\hline Q11 & -15 & 42 & 2.9300 & 5.002 \\
\hline Q13 & -15 & 48 & 8.1025 & 6.033 \\
\hline V3 & 0.95 & 1.05 & 1.0372 & 1.0392 \\
\hline V4 & 0.95 & 1.05 & 1.0307 & 1.0328 \\
\hline V6 & 0.95 & 1.05 & 1.0282 & 1.0298 \\
\hline V7 & 0.95 & 1.05 & 1.0101 & 1.0152 \\
\hline V9 & 0.95 & 1.05 & 1.0462 & 1.0412 \\
\hline V10 & 0.95 & 1.05 & 1.0482 & 1.0498 \\
\hline V12 & 0.95 & 1.05 & 1.0400 & 1.0466 \\
\hline V14 & 0.95 & 1.05 & 1.0474 & 1.0443 \\
\hline V15 & 0.95 & 1.05 & 1.0457 & 1.0413 \\
\hline V16 & 0.95 & 1.05 & 1.0426 & 1.0405 \\
\hline V17 & 0.95 & 1.05 & 1.0382 & 1.0396 \\
\hline V18 & 0.95 & 1.05 & 1.0392 & 1.0400 \\
\hline V19 & 0.95 & 1.05 & 1.0381 & 1.0394 \\
\hline V20 & 0.95 & 1.05 & 1.0112 & 1.0194 \\
\hline V21 & 0.95 & 1.05 & 1.0435 & 1.0243 \\
\hline V22 & 0.95 & 1.05 & 1.0448 & 1.0396 \\
\hline V23 & 0.95 & 1.05 & 1.0472 & 1.0372 \\
\hline V24 & 0.95 & 1.05 & 1.0484 & 1.0372 \\
\hline V25 & 0.95 & 1.05 & 1.0142 & 1.0192 \\
\hline V26 & 0.95 & 1.05 & 1.0494 & 1.0422 \\
\hline V27 & 0.95 & 1.05 & 1.0472 & 1.0452 \\
\hline V28 & 0.95 & 1.05 & 1.0243 & 1.0283 \\
\hline V29 & 0.95 & 1.05 & 1.0439 & 1.0419 \\
\hline V30 & 0.95 & 1.05 & 1.0418 & 1.0397 \\
\hline & & & & \\
\hline
\end{tabular}


Table 5: Comparison of Real Power Loss

\begin{tabular}{|l|l|}
\hline \multicolumn{1}{|c|}{ Method } & Minimum loss (MW) \\
\hline Evolutionary programming [32] & 5.0159 \\
\hline Genetic algorithm [33] & 4.665 \\
\hline Real coded GA with Lindex as SVSM [34] & 4.568 \\
\hline Real coded genetic algorithm [35] & 4.5015 \\
\hline Proposed HA method & 4.2989 \\
\hline
\end{tabular}

\section{Conclusion}

In this paper, proposed HA has been successfully implemented to solve optimal reactive power dispatch (ORPD) problem. The main advantages of HA when applied to the ORPD problem is optimization of different type of objective function, i.e real coded of both continuous and discrete control variables, and without difficulty in handling nonlinear constraints. Proposed HA algorithm has been tested on the IEEE 30-bus system. Simulation Results clearly show the good performance of the proposed algorithm in reducing the real power loss and enhancing the voltage stability.

\section{References}

[1] O.Alsac,and B. Scott, "Optimal load flow with steady state security",IEEE Transaction. PAS 1973, pp. 745-751.

[2] Lee K Y ,Paru Y M , Oritz J L -A united approach to optimal real and reactive power dispatch , IEEE Transactions on power Apparatus and systems 1985: PAS-104 : 1147-1153

[3] A.Monticelli , M .V.F Pereira and S. Granville, "Security constrained optimal power flow with post contingency corrective rescheduling", IEEE Transactions on Power Systems :PWRS-2, No. 1, pp.175-182.,1987.

[4] Deeb N ,Shahidehpur S.M ,Linear reactive power optimization in a large power network using the decomposition approach. IEEE Transactions on power system 1990: 5(2) : 428-435

[5] E. Hobson ,'Network consrained reactive power control using linear programming, ' IEEE Transactions on power systems PAS -99 (4) ,pp 868=877, 1980

[6] K.Y Lee ,Y.M Park, and J.L Oritz, "Fuel -cost optimization for both real and reactive power dispatches", IEE Proc; 131C,(3), pp.85-93.

[7] M.K. Mangoli, and K.Y. Lee, "Optimal real and reactive power control using linear programming", Electr.Power Syst.Res, Vol.26, pp.1-10,1993.

[8] C.A. Canizares, A.C.Z.de Souza and V.H. Quintana , "Comparison of performance indices for detection of proximity to voltage collapse,", vol. 11. no.3 , pp.1441-1450, Aug 1996 .

[9] Berizzi.C.Bovo,M.Merlo,andM.Delfanti,(2012), "A GA approach to compare ORPF objective functions including secondary voltage regulation," Electric Power Systems Research, vol. 84, no. 1 , pp. $187-194$.

[10] D. Devaraj, and B. Yeganarayana, "Genetic algorithm based optimal power flow for security enhancement", IEE proc-Generation.Transmission and. Distribution; 152, 6 November 2005.

[11] A.Berizzi, C. Bovo, M. Merlo, and M. Delfanti, "A ga approach to compare orpf objective functions including secondary voltage regulation," Electric Power Systems Research, vol. 84, no. 1, pp. $187-194,2012$.

[12] C.-F. Yang, G. G. Lai, C.-H. Lee, C.-T. Su, and G. W. Chang, "Optimal setting of reactive compensation devices with an improved voltage stability index for voltage stability 
enhancement," International Journal of Electrical Power and Energy Systems, vol. 37, no. 1, pp. $50-57,2012$.

[13] P. Roy, S. Ghoshal, and S. Thakur, "Optimal var control for improvements in voltage profiles and for real power loss minimization using biogeography based optimization," International Journal of Electrical Power and Energy Systems, vol. 43, no. 1, pp. 830 - 838, 2012.

[14] B. Venkatesh, G. Sadasivam, and M. Khan, "A new optimal reactive power scheduling method for loss minimization and voltage stability margin maximization using successive multi-objective fuzzy lp technique," IEEE Transactions on Power Systems, vol. 15, no. 2, pp. 844 - 851, may 2000.

[15] W. Yan, S. Lu, and D. Yu, "A novel optimal reactive power dispatch method based on an improved hybrid evolutionary programming technique," IEEE Transactions on Power Systems, vol. 19, no. 2, pp. $913-918$, may 2004.

[16] W. Yan, F. Liu, C. Chung, and K. Wong, "A hybrid genetic algorithminterior point method for optimal reactive power flow," IEEE Transactions on Power Systems, vol. 21, no. 3, pp. 1163 1169, aug. 2006.

[17] J. Yu, W. Yan, W. Li, C. Chung, and K. Wong, "An unfixed piecewiseoptimal reactive powerflow model and its algorithm for ac-dc systems," IEEE Transactions on Power Systems, vol. 23, no. 1, pp. $170-176$, feb. 2008.

[18] F. Capitanescu, "Assessing reactive power reserves with respect to operating constraints and voltage stability," IEEE Transactions on Power Systems, vol. 26, no. 4, pp. 2224-2234, nov. 2011.

[19] Z. Hu, X. Wang, and G. Taylor, "Stochastic optimal reactive power dispatch: Formulation and solution method," International Journal of Electrical Power and Energy Systems, vol. 32, no. 6, pp. $615-621,2010$.

[20] A.Kargarian, M. Raoofat, and M. Mohammadi, "Probabilistic reactive power procurement in hybrid electricity markets with uncertain loads," Electric Power Systems Research, vol. 82, no. 1, pp. $68-80,2012$.

[21] J. H. Holland. "Outline for a logical theory of adaptive systems". Journal of the ACM, 3:297314, 1962.

[22] Talbi, El-Ghazali. "Metaheuristics: from design to implementation". Vol. 74. John Wiley \& Sons, 2009.

[23] Bansal, J. C. et al. "Information Sharing Strategy among Particles in Particle Swarm Optimization Using Laplacian Operator”, Swarm Intelligence Symposium, 2009. IEEE, pages 30-36.

[24] Wright, "A. Genetic Algorithms for Real Parameter Optimization, Foundations of Genetic Algorithms", G. Rswlins(Ed.), Morgen Kaufmann publishers, CA, 1991, pp. 205-218.

[25] Yu-hui Shi and Russell Eberhart," A modified particle swarm optimizer," in proceedings of IEEE world Congress on Computation intelligence,pp.69-73,1998.

[26] Takahashi, Masato, and Hajime Kita. "A crossover operator using independent component analysis for real-coded genetic algorithms." Evolutionary Computation, 2001. Proceedings of the 2001 Congress on. Vol. 1. IEEE, 2001.

[27] Zhi-Feng Hao,Zhi-Gang Wang,HanHuang,A Particle Swarm Optimization Algorithm With Crossover Operator Proceedings of the 6th international conference on Machine Learning and Cybernatics ,Hong Kong ,19-22 August 2007.

[28] Chen, Stephen. "Particle swarm optimization with pbest crossover."Evolutionary Computation (CEC), 2012 IEEE Congress on. IEEE, 2012[23]

[29] X.S. Yang. A new metaheuristic bat-inspired algorithm. Nature Inspired Cooperative Strategies for Optimization (NICSO 2010), pages 65-74, 2010.

[30] A.H. Gandomi, X.S. Yang, A.H. Alavi, and S. Talatahari. Bat algorithm for constrained optimization tasks. Neural Computing \& Applications, pages 1-17, 2012.

[31] P.W. Tsai, J.S. Pan, B.Y. Liao, M.J. Tsai, and V. Istanda. Bat algorithm inspired algorithm for solving numerical optimization problems. Applied Mechanics and Materials, 148:134-137,2012. 
[32] Wu Q H, Ma J T. "Power system optimal reactive power dispatch using evolutionary programming", IEEE Transactions on power systems 1995; 10(3): 1243-1248 .

[33] S.Durairaj, D.Devaraj, P.S.Kannan "Genetic algorithm applications to optimal reactive power dispatch with voltage stability enhancement", IE(I) Journal-EL Vol 87,September 2006.

[34] D.Devaraj , "Improved genetic algorithm for multi - objective reactive power dispatch problem", European Transactions on electrical power 2007 ; 17: 569-581.

[35] P. Aruna Jeyanthy and Dr. D. Devaraj "Optimal Reactive Power Dispatch for Voltage Stability Enhancement Using Real Coded Genetic Algorithm", International Journal of Computer and Electrical Engineering, Vol. 2, No. 4, August, 2010 1793-8163.

*Corresponding author.

E-mail address: gklenin@ gmail.com 